\title{
COMPUTATIONAL TOPOLOGY FOR CONFIGURATION SPACES OF HARD DISKS
}

\author{
GUNNAR CARLSSON, JACKSON GORHAM, MATTHEW KAHLE, AND JEREMY MASON
}

\begin{abstract}
We explore the topology of configuration spaces of hard disks experimentally, and show that several changes in the topology can already be observed with a small number of particles. The results illustrate a theorem of Baryshnikov, Bubenik, and Kahle that critical points correspond to configurations of disks with balanced mechanical stresses, and suggest conjectures about the asymptotic topology as the number of disks tends to infinity.
\end{abstract}

\section{INTRODUCTION}

Configuration spaces of hard spheres are of intrinsic interest mathematically and physically, yet so far little seems known about their topology. As shown in this note, the dependence on the volume fraction (or equivalently on the sphere radius) of properties as fundamental as the connectivity of configuration space is already quite complicated even for a small number of spheres. More generally, we are concerned not only with the property of connectivity or with the number of connected components $\beta_{0}$, but also with the $i$ th Betti number $\beta_{i}$ which denotes the dimension of $i$ th homology, or colloquially "the number of $i$-dimensional holes [1]."

One purpose of this article is to illustrate some of the complexity of the topology of configuration spaces of hard spheres, even in two dimensions (i.e. hard disks) when the number of disks is small. We illustrate a theorem of Baryshnikov, Bubenik, and Kahle that mechanically-balanced (or jammed) configurations act as critical points, indicating the only places where the topology can change [2]. We always mean "critical point" in the mathematical sense of non-differentiable or singular, rather than the statistical mechanical sense. Examples of such configurations may be seen in Figure 1. The combinatorial and geometric structure of the bond networks of these critical points is important in classifying their degeneracy and in computing their index. Furthermore, the bond networks suggest connections to the geometric structure approach in hard particle packing [3], as well as to tensegrity and strut frameworks [4].

We show here that the topology of these configuration spaces can already be fairly complicated in two dimensions with a small number of particles. For example, with five disks in the unit square, the topology changes at least 20 times as the disk radius varies, and for radius $0.1686<r<0.1692$ the configuration space has $\beta_{1}=2176$ "holes".

The topology of configuration spaces has been studied in the context of motion planning for robots; see the surveys by Farber and Ghrist [5, 6], and the textbook by LaValle [7. One central idea in this field is that topological complexity, measured

Date: October 29, 2018.

MK was supported in part by NSA Grant \# H98230-10-1-0227. 

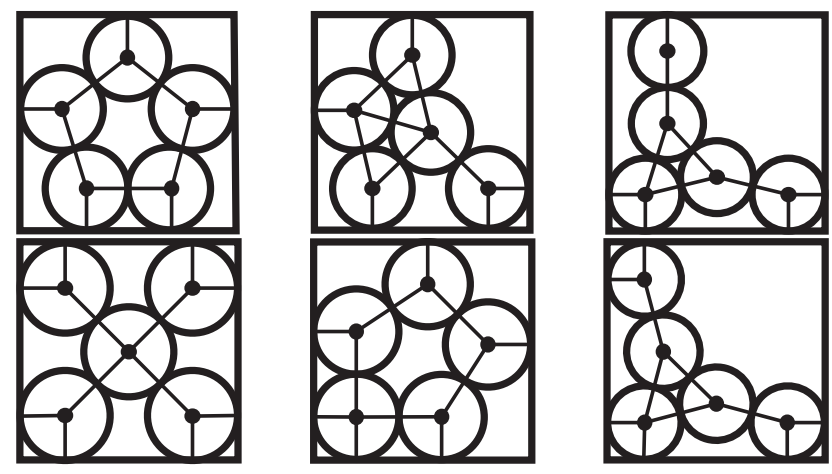

Figure 1. Index zero (bottom row) and index one (top row) critical points, shown with their bond networks.

in various ways, is a measure of the difficulty of constructing a motion planning algorithm. Many papers idealize robots as point particles in a suitable metric space - although it has been noted that the assumption that they have no volume is not physically realistic [8]. On the other hand, expanding the particles to have positive thickness greatly complicates the topology of the underlying configuration space see for example the recent work of Deeley on thick particles on a metric graph [9].

As further motivation for this study, a set of hard disks inhabiting a bounded area may be regarded as a model thermodynamic system. From this standpoint, a topological property such as connectivity of the configuration space is a fundamental concern, since this is required to satisfy the assumptions of equal a priori probabilities and ergodicity (e.g., Section VII of Torquato and Stillinger [3]) inherent to the statistical mechanics formalism. Despite the importance of this question, few results seem to be known for finite hard disk systems [10, and fewer still in the thermodynamic limit. It is known that if one can only move one disk at a time, the configuration space for large numbers of disks can be disconnected even for density arbitrarily close to zero [11, 12. Even if collective motions of disks are allowed, the configuration space may be disconnected for density bounded away from maximal - for example, the reinforced Kagome lattice is strictly jammed and only has density $\approx 0.68$ compared to the close packing density of $\approx 0.91[13$.

Perhaps one of the most notable properties of the hard disk system in the context of thermodynamics is the existence of a solid-to-liquid phase transition [14] with the variation of the packing fraction, or equivalently of the disk radius. This is particularly suggestive in the context of a number of recent papers that explore the hypothesis that statistical-mechanical phase transitions are intimately related to changes in the topology of surfaces of constant potential (i.e. equipotential submanifolds) in the configuration space [15, 16, 17, 18. Specifically, a theorem of Franzosi, Pettini, and Spinelli [19, 20] states that for rather benign conditions (smooth, finite-range, confining potentials), the low-order derivatives of the Helmholtz free energy cannot display a discontinuity unless there is simultaneously a change in the topology of the equipotential submanifolds with the variation of a relevant parameter, such as the disk radius. While this indicates that a topological change is necessary, a conjecture known as the Topological Hypothesis roughly states that a major topological change (a proposal for specific conditions is given by 
Kastner, Schreiber and Schnetz [21, 22]) is sufficient to drive the phase transition as well. This immediately raises the question of the topological signature of the solid-to-liquid phase transition for the hard disk system.

Historically, the accumulation of certain indirect evidence encouraged the development of the Topological Hypothesis. For example, the magnitude of fluctuations in the curvature of equipotential submanifolds was observed to show singular behavior near a phase transition 23. This singular behavior seemed to be insensitive to the choice of metric [24, pointing to a more fundamental change in the topology of the accessible portion of configuration space at the phase transition. Further support was provided by the existence of major changes in the topology of the equipotential submanifolds accompanying phase transitions in certain exactly solvable systems $25,26,16,17$. Strikingly, the presence of these changes is known to persist for small systems well away from the thermodynamic limit 25, 27]. That is, a major topology change in the equipotential submanifolds of a system with just a few hard disks may indicate the presence of a topology change that drives the phase transition of the corresponding system in the thermodynamic limit.

There is a persistent difficulty faced by investigations of the Topological Hypothesis in the physics literature, namely, that the exactly solvable systems mentioned above do not satisfy the conditions for the theorem of Franzosi, Pettini and Spinelli. Specifically, they violate the requirements of short-range interactions [25, 26, 17. and confining potentials [16. This stands in sharp contrast to the short-range, hard-core repulsive potential characteristic of hard disks systems, meaning that a complete characterization of the configuration space topology of our system will begin to address a need that is openly acknowledged in the literature [17.

We begin this study by reconstructing the dendrogram, i.e. a graph which encodes the appearance of path components and the connections between them as the disk radius (or volume fraction) varies. More generally, for a suitably defined energy function, the dendrogram is defined to be the graph with nodes given by the basins of attraction in the energy landscape and edges given by the saddle points that connect the basins as the energy increases. These correspond in the physics literature to collectively-jammed configurations and to the transitions between them (see Torquato \& Stillinger [3]), subjects of continuing interest to the physics community. The name "dendrogram" comes from hierarchical clustering in statistics. We find the saddle points using the "nudged elastic band" method [28]. From the point of view of Morse theory, the index of the basins of attraction is zero since there is no collective motion that decreases the energy, and the index of the saddle points is one since there is one dimension of collective motion that decreases the energy. Examples of these varieties of critical points appear in Figure 1.

As alluded to above, these features of the configuration space correspond in the Morse-theory literature to critical points of index zero and index one, respectively 29]. Along with the notion of a critical index comes the notion of higher index critical points, allowing for the construction of a more complete picture of the configuration space and the related energy landscape. This is precisely the field of Morse theory, which provides a framework to pass from the populations of critical points with specified indices to a reconstruction of the configuration space, and allows us to answer many more questions than simply the energy at which the saddle point connecting jammed configurations is accessible. For instance, we deduce certain facts about the Betti numbers $\beta_{k}$ that indicate the number of $k$-dimensional 
holes or the dimension of $k$ th homology, and observe suggestive patterns about the asymptotic topology of configuration spaces for large numbers of disks.

There is good reason to introduce Morse theory as a fundamental instrument for the study of configuration spaces. Various flavors of Morse theory sit at the heart of Franzosi, Pettini, and Spinelli's proof [19] in the context of the Topological Hypothesis, Yao et al.'s exploration of low-density states in biomolecular folding pathways 30, and Deeley's study of configuration spaces of thick particles on a metric graph [9]. Section 3 will briefly overview Morse theory, though most of the technical mathematical details, and particularly the proof of the main theorem we quote, are left to another article 2]. Our primary focus in this article is on our numerical experiments to identify critical points of various indices and connecting the Morse theoretic viewpoint of configurations spaces with related concepts in the physics literature.

\section{Configuration spaces}

Let $\mathbf{C}(n, r)$ denote the configuration space of $n$ nonoverlapping disks of radius $r$ in the unit square $[0,1]^{2}$. If we indicate the coordinates of the center of the $i$ th disk by $x_{i}$, we can write this as

$$
\mathbf{C}(n, r)=\left\{\left(x_{1}, x_{2}, \ldots, x_{n}\right) \mid x_{i} \in[r, 1-r]^{2}, d\left(x_{i}, x_{j}\right) \geq 2 r\right\} .
$$

Since $\mathbf{C}(n, r) \subset \mathbb{R}^{2 n}$, it naturally inherits a subspace topology and a metric from the Euclidean space of the appropriate dimension. The configuration space of hard disks in the limit of large $r$ is the empty space, since the disks do not fit in the unit square. Meanwhile, the configuration space of $n$ labelled points in the plane (or more generally on a manifold) is well studied [31, 32, and is equivalent to the configuration space of hard disks in the limit of small $r$. This leaves the study of the topology of $\mathbf{C}(n, r)$ for intermediate $r$, which is much more complicated than for the two cases above.

Notice that if $r^{\prime}<r$, then there exists a natural inclusion map

$$
\mathbf{C}(n, r) \hookrightarrow \mathbf{C}\left(n, r^{\prime}\right)
$$

given by shrinking the disks with fixed centers. In other words, as the disk radius shrinks, the space of allowable configurations grows.

As mentioned in Section 1 a basic question is whether $\mathbf{C}(n, r)$ is connected for a given value of $r$, and if not, how many components there are. A more subtle description involves the construction of the dendrogram, or the graph that encodes the appearance of persistent components and the connections between them as the disk radius varies. Equivalently, the dendrogram is a means to visualize distinct basins of attraction in the energy landscape and the saddle points between them, not the properties of the configuration space at higher energies. That would require identifying the critical points of all indices and using Morse theory to reconstruct the remaining features of the configuration space.

We give a brief discussion of Morse theory to establish a unifying set of language and ideas. Following that, we apply each of these approaches to the specific case of the configuration space of five disks in the unit square.

\section{Morse THEORY}

The basic idea of Morse theory is to filter a topological space $X$ by the sublevel sets $f^{-1}((-\infty, r])$ of a suitable function $f: X \rightarrow \mathbb{R}[29$, 33]. Suppose that 
the function $f$ assigns an energy $E$ to every point in the configuration space, and that this energy increases as the radius of the disks decreases. The sub-level set associated with a particular energy $E_{0}$ is just the set of accessible states, or the set of points in the configuration space where the energy is less than or equal to $E_{0}$. The existence of the inclusion map in the preceding section is equivalent to the statement that as $E_{0}$ increases, the set of accessible states increases. Provided that the function $f$ is well-behaved, the topology of the sub-level sets will change only a small number of times as $r$ decreases. One of the tenets of traditional Morse theory is that these changes occur precisely at the critical points where $\nabla f(x)=0$, or at the mechanical equilibria of the system. Using techniques similar to those in "Mintype" Morse theory [34] and stratified Morse theory [35], Baryshnikov, Bubenik, and Kahle recently proved the following theorem [2].

Theorem 3.1. If there are no "balanced" configurations of disks of radius $r$ with $r \in[a, b]$ then $\mathbf{C}(n, a)$ is homotopy equivalent to $\mathbf{C}(n, b)$.

The definition of a "mechanically-balanced" configuration is as follows. For a configuration of disks, define the bond network to have vertices at disk centers, and edges $e_{i j}$ connecting pairs of tangent disks $\{i, j\}$, and also disks to the boundary of the region. We consider the edge $e_{i j}$ to be a vector, pointing from $i$ to $j$, so in particular $e_{i j}=-e_{j i}$.

Definition 3.2. A configuration is mechanically-balanced if non-negative weights $w_{i j}$ can be assigned to the edges of the bond network so that at least one $w_{i j}$ is positive, and for every fixed vertex $i$,

$$
\sum_{j \sim i} w_{i j} e_{i j}=0 .
$$

where the sum is over all vertices $j$ adjacent to $i$.

The point here is that $\mathbf{C}(n, r)$ is the sub-level sets of some function - but not a smooth function. Smooth Morse theory and Morse-Bott theory don't quite work in this case, but something similar to min-type Morse theory 34 does work. We note that this is similar in spirit to the PL Morse-Bott Theory in Deeley's recent work 9 .

For a smooth Morse function the index of a non-degenerate critical point is the number of negative eigenvalues of the Hessian. For a min-type Morse function (such as for hard disks), you can compute the index of critical points with linear programming, i.e. the index is the dimension of a certain cone. In either form of Morse theory, the fundamental theorem states that every time you have an index $k$ critical point, the homotopy type changes by gluing in another $k$-dimensional cell, and importantly - that there are not any other changes in the topology except when there are critical points.

\section{Part I: Dendrogram}

It is convenient for our methods to first slightly "soften" the disks. We recover their hardness shortly. In general, on the configuration space of $n$ distinct points in a region $R$ we can define a smooth energy function $E$ by

$$
E=\sum_{1 \leq i<j \leq n} \frac{1}{\left(d\left(x_{i}, x_{j}\right) / 2\right)^{h}}+\sum_{1 \leq i \leq n} \frac{1}{d\left(x_{i}, \partial R\right)^{h}}
$$


where $h \gg 0$ is a fixed "hardness" parameter and $\partial R$ denotes the boundary of $R$. For our experiments we set $h=50, n=5$, and $R=[0,1]^{2}$.

Taking uniform random points in $[0,1]^{10}$ and flowing in the direction of steepest descent along $-\nabla E$, we find the three qualitatively different types of local minima illustrated in the bottom row of Figure 1. We believe this to be a complete list of all "types" of local minima (see discussion below) for five disks in a box.

Notice that a point in $[0,1]^{10}$ gives the coordinates of five distinguishable disks. Since exchanging the labels of the disks does not change the energy of the configuration, this permutation symmetry entails that every critical point be symmetrically equivalent to 120 points in the configuration space (including itself). (To mathematicians, the labeled configuration space admits a free action of the symmetric group, and the quotient is the unlabeled configuration space.) This number of instances of each type is further increased by the additional configurations generated by applying the symmetries of the unit square. For instance, the class of configurations symmetrically equivalent to the critical point at a radius of 0.2071 in Figure 2 contains 120 points, while the equivalence class containing the critical point at a radius of 0.1964 contains 480 points, and the equivalence class containing the critical point at a radius of 0.1693 contains 960 points. So by a "type" of critical point, we mean an equivalence class of critical points under both types of symmetries.

Once a local minimum of $E$ is found, the preliminary radius $r$ of the configuration is the maximum possible radius such that that no disks overlap or extend outside the unit square. The "bond network" of the configuration is found by connecting centers of disks that are within $(2+\epsilon) r$ of each other, or within $(1+\epsilon) r$ of the boundary. Here $\epsilon>0$ is a fixed small tolerance parameter, usually around 0.001. With the bond network established, we effectively harden the disks by searching for an exact solution to the set of equations given by varying $r$ and setting $\epsilon=0$. In some cases this gives a unique solution, and establishes the final values of the radius for the three red configurations along the bottom of Figure 2

We then consider pairs of local minima which are close in the configuration space for the induced metric and find low energy paths between them using the "nudged elastic band" method [36, 28. The highest energy points along a low energy path should be close to a saddle point. Indeed, by establishing the bond networks for all of the highest energy points along low energy paths and applying the hardening procedure described above, we identify the saddle points depicted in orange along the bottom of Figure 2. This allows us to construct the dendrogram for our system, though a full graphical representation of this graph would be quite complicated. We present simplified versions of some of the salient features in Figures 2,4 and 5.

A precise value of the radius is important to be able to order the critical points by the radius of the disks when they appear. Evidence that a rough numerical approach is not sufficient is provided by the two critical points of index one in Figure 3. where the radius differs only in the fourth decimal place. This is one reason why the more elaborate approach described above is necessary.

Figure 2 shows the variation in the number of connected components as a function of the radius. The total number of connected components for a given radius is shown at the top of the figure. The central part of the figure collects the connected components into classes of symmetrically equivalent elements, and labels the classes 


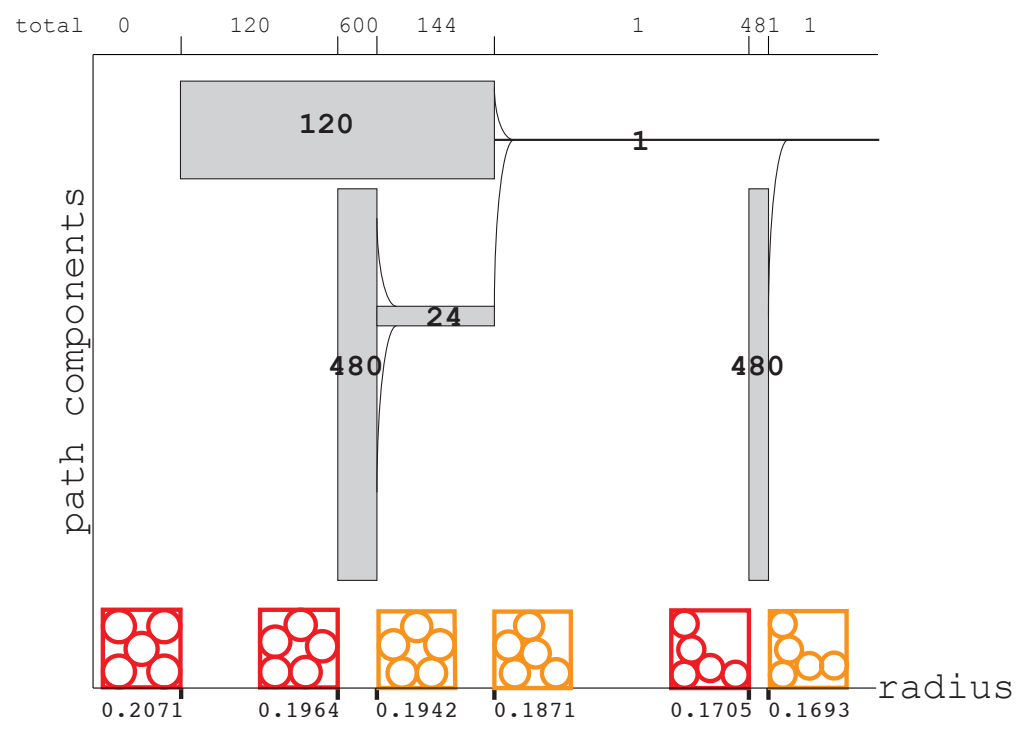

Figure 2. (Color online.) A bird's-eye view of the dendrogram for labeled disks. New components appear at radius 0.2071, 0.1964, and 0.1705 , and components merge together at radius 0.1942 , 0.1871 , and 0.1693 .

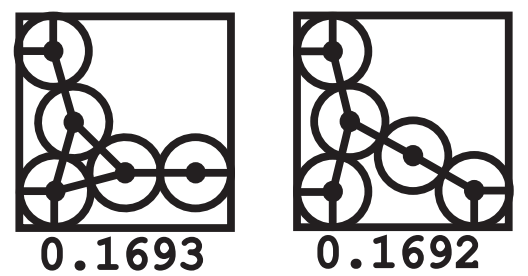

FiguRE 3. Two saddle points with nearly the same radius

by the number of elements they contain. The bottom of the figure shows the configurations of the disks at the relevant critical points, and provides the values of the radius when these configurations appear.

For $r>0.2071$, the disks do not fit inside the unit square and the configuration space is empty. The first allowed configurations occur at a radius of 0.2071 , when 120 connected components appear simultaneously. All of these belong to the same class, and correspond to different labelings of the disks in the first red configuration. As the radius decreases, a class of 480 more connected components appear at radius 0.1964, corresponding to different labelings and arrangements of the disks in the second red configuration. The 480 components of this class connect in groups of 20 with the emergence of the first saddle point at radius 0.1942 , leaving a class of 24 embedded circles. One of these circles is shown in Figure 4. Every arc segment connecting the jammed configurations in Figure 4 is a low energy path containing a saddle point of the type appearing at radius 0.1942 . The appearance of the second saddle point at radius 0.1871 connects all of the remaining components. The space remains connected for all smaller values of the radius, with the exception of the 


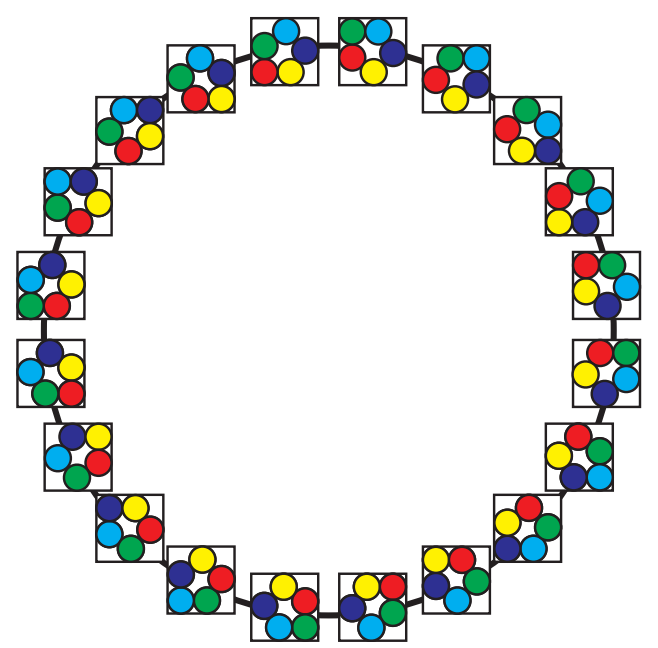

FiguRE 4. (Color online.) For radius $0.1871<r<0.1942$, each connected component of the configuration space is homotopy equivalent to a circle. One such embedded circle and 20 index-0 critical points is shown here. Not shown are the 20 transitionary index-1 critical points between them.

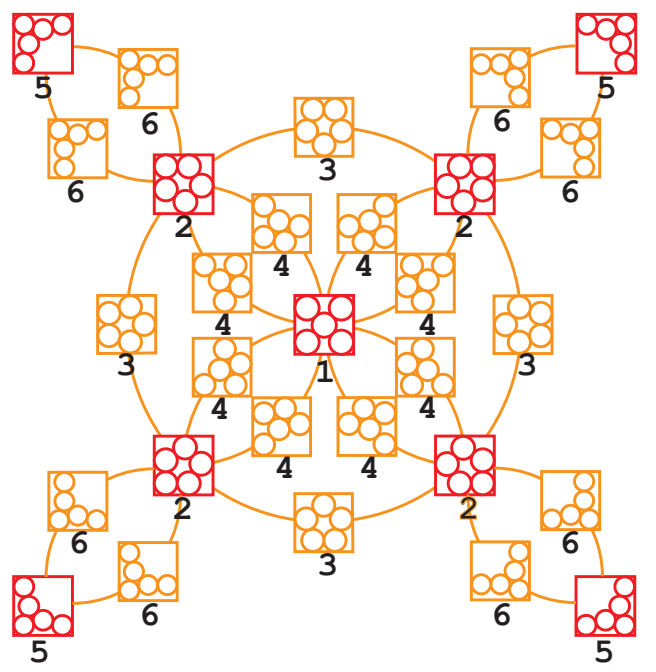

Figure 5. (Color online.) The dendrogram for unlabeled disks. The numbers indicate the order in which critical points appear as the disks shrink - new components appear at 1,2, and 5, and components merge at 3,4 , and 6 . The radius where each vertex and edge appear is given in Figure 8.

appearance of 480 more components at a radius of 0.1705 , though these connect up with the main component soon afterward. 
An alternate representation of the dendrogram is given in Figure 5 . Since the purpose of this figure is to allow the visualization of the paths connecting energy minima, we consider only unlabeled disks for the sake of simplicity. The labels of the critical points indicate the order of appearance as the radius of the disks decreases, where a set of critical points with the same labels constitutes an equivalence class. Similar to the description in Figure 2, the first allowed configuration to appear is the red critical point in the center of the figure. As the radius decreases this single configuration is joined by the four critical points arrayed around the central circle, though they all remain isolated until the first saddle points appear. At this interval the configuration space is given by the isolated configuration in the center and a circle that is roughly analogous to that in Figure 4 , but with unlabeled disks. The central configuration and the circle become connected with the appearance of the second saddle point, and the space remains a single component until the final energy minima appear at the outer extremities of the figure. The third saddle point once more connects the space into a single component.

Figures 2 and 5 do not show all of the critical points of index one for five disks in the unit square. Indeed, we identified five types of critical points of index one, though only three of these change the number of connected components in the configuration space. Figures 2 and 5 show precisely these three types of critical points, since the remaining two do not directly affect the connectivity of the space.

\section{PART II: Higher index CRITICAL POINTS}

While the above section is concerned with the critical points of index zero and index one and the relation of these to the dendrogram, the current section is concerned with more general features of the configuration space. As mentioned in Section 1, this requires the identification of the critical points of higher index. For this purpose, we work with the same smooth function $E$ defined above. Using the MuPAD computer algebra system included in the Symbolic Math Toolbox for MATLAB, we symbolically differentiate $E$ and set

$$
F=\|\nabla E\|^{2} \text {. }
$$

Since $\nabla E=0$ at the critical points of $E, F=0$ at the critical points of $E$ as well and is strictly greater than zero everywhere else. Hence, we may find critical points of $E$ of any index by flowing down the function $F$ to one of the zeros.

More specifically, our search begins from a random point in $[0,1]^{10}$. We symbolically calculate the gradient of $F$ with MuPAD and apply the conjugate gradient method with the Polak-Ribière formula, using the secant method to find the minimum of $F$ in the search direction on every iteration. This gives much more rapid convergence to the local minima of $F$ than the steepest descent algorithm.

Once the conjugate gradient algorithm converges, the preliminary radius $r$ of the configuration is the maximum possible radius such that that no disks overlap or extend outside the unit square. The bond network is constructed just as for the calculation of the dendrogram, though the tolerance parameter $\epsilon$ is on the order of 0.01 . We recover the configuration of the critical point in the hard disk system by modeling the bonds in the bond network as springs. Let $s$ be the average of the bond lengths from the disks to the boundary and half the bond lengths connecting pairs of disks. Set the equilibrium length of the springs from the disks to the boundary to be $s$, and the equilibrium length of the springs connecting pairs of 


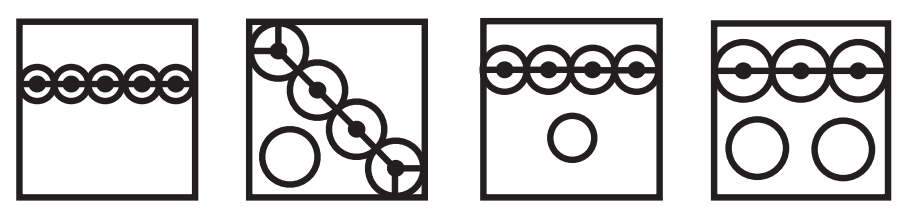

Figure 6. From left to right, critical submanifolds of dimensions $1,2,3$, and 5 .

disks to be $2 s$. We minimize the potential energy of the spring network using the conjugate gradient method and update $s$ on every iteration. This is repeated while reducing the spring constant from a small positive number to zero, allowing us to find the radius of the disks at a critical point of the hard disk system to more than five significant digits.

The index of a critical point is found by symbolically calculating the Hessian of $E$, evaluating this expression for the given disk locations, and numerically finding the number of negative eigenvalues. Provided that the Hessian is well-conditioned, the index of a critical point calculated for the smooth function $E$ should be the same as for the hard disk system. Some discretion should be used when finding the critical index this way though, since the Hessian is not always well-conditioned. In these cases, the index is found as the dimension of a certain cone calculated from the bond network.

We found only 30 types of critical points and critical submanifolds after repeating this process on the order of $10^{5}$ times. By "critical submanifold" we mean a critical point with several degrees of freedom, where the number of degrees of freedom is the dimension of the submanifold. (In the strict mathematical sense, these might not be manifolds or even manifolds with boundary.) Specifically, the presence of each "rattler" contributes two dimensions to the critical submanifold, while a row of disks across the square adds one dimension. Several examples of these appear in Figure 6. along with the corresponding dimensions. Notice that the 5-dimensional submanifold on the right is not contractible, as the others are - this one is homotopy equivalent to a circle.

Of these 30 types of critical points and submanifolds, we consider the 10 in Figure 7 to be degenerate. Our criterion is if weights $w_{i j}$ may be assigned to the edges of the bond network of a balanced configuration so that at least one $w_{i j}$ is positive and at least one $w_{i j}$ vanishes, then the critical point or submanifold is degenerate. Computing the local topology near several critical points that satisfy this condition has convinced us that the topology does not change at these critical points, but we do not yet state this as a formal theorem. We intend to explore the question of degeneracy in more mathematical detail in a future paper.

The remaining 20 non-degenerate critical points and submanifolds appear in Figure 8 , ordered horizontally by the radius of the disks and vertically by index.

This figure reveals that higher index critical points generally coincide with smaller radius. For instance, all of the index- 0 and index- 1 critical points occur at larger radius than any of the higher-index critical points. The fundamental theorem in Morse theory states that each critical point of $i$ contributes an $i$-dimensional cell via an attaching map, up to homotopy equivalence (smooth deformation) [35, 29. This indicates that for radius $r \geq 0.1692$, the whole configuration space is homotopy equivalent to a 1-dimensional cell complex, i.e. a graph. 

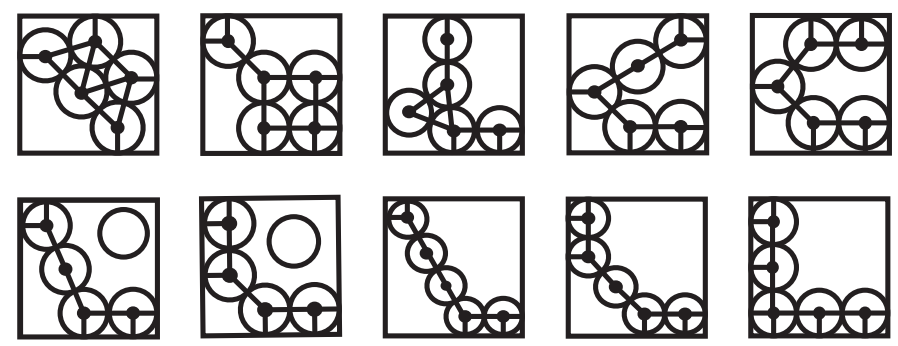

FIGURE 7. Degenerate types
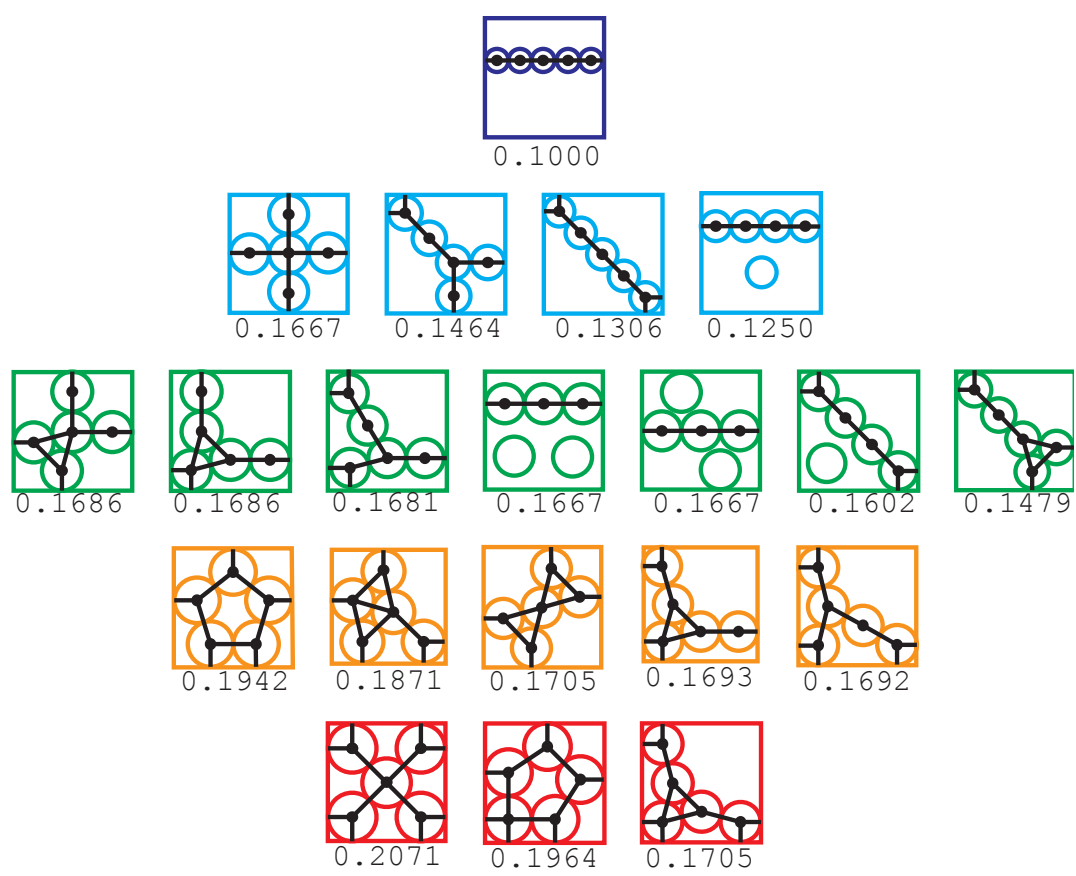

Figure 8. (Color online.) Non-degenerate types, in rows by index. Index 0 critical points are in the bottom row, all the way up to index 4 in the top row.

The index- 0 critical points correspond to vertices of this graph, and the index- 1 critical points correspond to edges. Since we have already computed the number of connected components $\beta_{0}$ in the dendrogram above, the Euler relation

$$
v-e=\beta_{0}-\beta_{1} .
$$

allows us to compute $\beta_{1}$ - the results in Figure 9. While the topology changes from the two critical points with the same radius $r \approx 0.1705$ are simultaneous, their indices are distinct and their contribution to the Betti numbers are computed separately. The calculation does not continue for $r<0.1686$ since we only have bounds on the Betti numbers after this point - with the attachment of 2-cells, the Euler relation

$$
v-e+f=\beta_{0}-\beta_{1}+\beta_{2}
$$




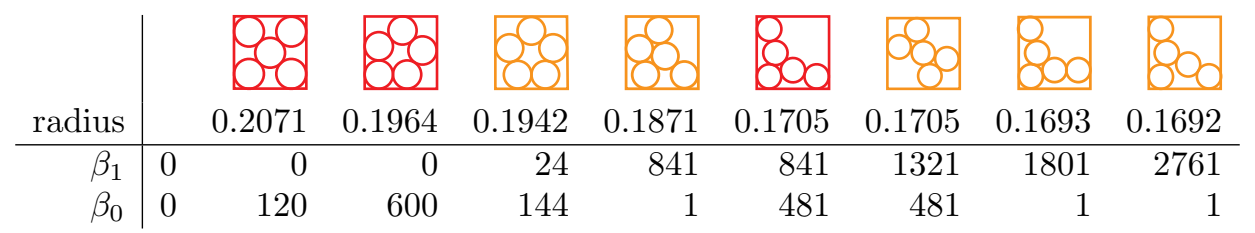

Figure 9. (Color online.) Betti numbers for the first several changes in topology.

alone is not sufficient to find $\beta_{1}$.

From Figure 9 we see that for $0.1686<r<0.1692$ the configuration space is homotopy equivalent to a bouquet of 2761 circles. Since we know that no more 1-cells are attached for $r<0.1692$, we have that $\beta_{1}$ is monotone decreasing as the radius decreases from this point on. Meanwhile, for small enough radius, $\beta_{1}=10$ since this is the Betti number for the configuration space of 5 points in the plane. Therefore, for five disks in the unit square we know that $\beta_{1}$ is a unimodal function of the radius and that its peak value is $\beta_{1}=2761$.

The non-degenerate critical points are shown sorted by radius (and in rows by index) in Figure 10. In this histogram, it is seen that all the critical points have radius between 0.100 and 0.220 - however half of them occur between radius 0.160 and 0.175 . This suggests the intriguing possibility that a large number of critical points are concentrated in a small interval as the number of particles $n \rightarrow \infty$, potentially the type of situation required by the Topological Hypothesis to drive a phase transition.

We do not know with certainty that we have a complete list of all types of critical points for 5 disks in a box - for example, it is easily possible that some points have very small basins of attraction for the algorithms we used, and that we also failed to find them by hand. If so, it would change the calculations we have made - nevertheless, we expect that we have most of them and one or two missing types would likely not significantly alter the qualitative behavior we observe.

\section{Comments}

It is probably within computational reach to implement experiments like these for greater numbers of disks, spheres in higher dimensions, and in other types of bounded regions. Flat tori and sphere are especially attractive boundaryless 2dimensional cases. Even if it is difficult to find a complete list of critical points for large numbers of disks, it would still be interesting to sample a large selection of them. It might be possible, for example, to find a geometrically feasible bond network and then increase the disk radius, preserving tangency at all time, until a critical point is found. Compiling statistics on radius and index might already give interesting information, as in Figure 10. New techniques for exhausting index0 jammed configurations, and over all possible lattices, are described in [37, and these methods could likely be adapted to higher index points.

A promising area for future study is the asymptotic topology of $\mathbf{C}(n, r)$ as $n \rightarrow$ $\infty$. It is generally not even known how large one can make $r=r(n)$ and maintain the property that $\mathbf{C}(n, r)$ is connected as $n \rightarrow \infty$. For ergodicity in a convex region, it is straightforward but important to ensure that the condition $r \leq C / n$ for some region-dependent constant $C$ implies that $\mathbf{C}(n, r)$ is connected [38, 39]. However, 


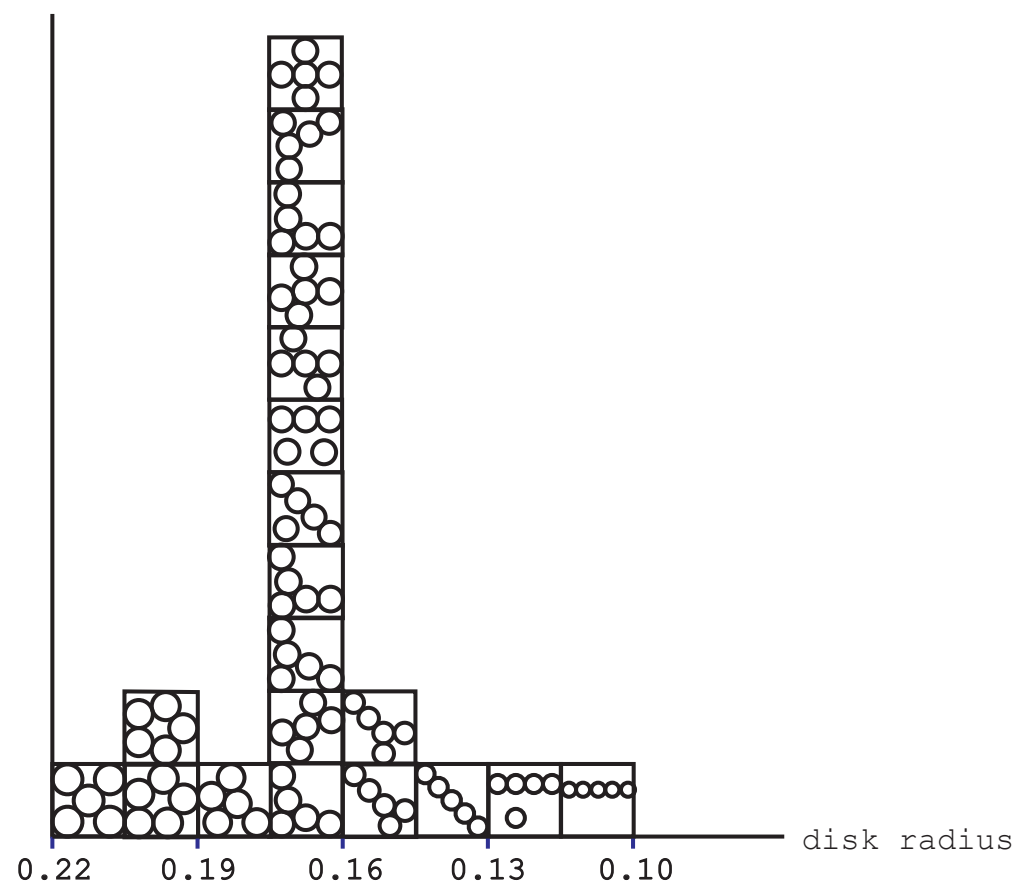

FiguRE 10. Histogram of the nineteen types of non-degenerate critical points

spheres satisfying this condition are quite small, taking up only length rather than volume in the limit, and it may be that some configuration spaces are connected for much larger radius.

The question of threshold radius for connectivity depends on the bounding region - the case of a circle implies that the $C / n$ bound is the best possible when the region is not constrained. It has been conjectured that for a flat torus though, the least dense collectively jammed configuration is the (reinforced) Kagomé lattice [13. If an analogue of this conjecture holds for index zero points and index one critical points, for example, then $r=O\left(n^{-1 / d}\right)$ would ensure connectivity of $\mathbf{C}(n, r)$.

A more general question is the asymptotic behavior of the Betti numbers when the radius is set to $r=r(n)$ and $n \rightarrow \infty$. We can guess but not yet prove several of the qualitative features of the asymptotic topology of $\mathbf{C}(n, r)$ for this situation. For example, we expect $\beta_{0}$ to be roughly decreasing with radius, and each Betti number $\beta_{k}$ with $k \geq 1$ to be roughly unimodal in the disk radius. In support of this conjecture, we display the Betti numbers we computed for four disks in a square in Figure 11 - in this case there are fewer changes in the topology, the index of a critical point is monotone decreasing in the radius, and we were able to compute all the Betti numbers.

\section{Acknowledgements}

We thank Yuliy Baryshnikov, Fred Cohen, Bob MacPherson, and Sal Torquato for helpful and inspiring conversations, and especially Persi Diaconis, who suggested studying the topology of these configuration spaces. JM and MK thank the Institute 


\begin{tabular}{|c|c|c|c|c|c|c|c|c|c|}
\hline$\beta_{3}$ & 0 & 0 & 0 & 0 & 0 & 0 & 0 & 0 & 6 \\
\hline$\beta_{2}$ & 0 & 0 & 0 & 0 & 0 & 0 & 5 & 53 & 11 \\
\hline$\beta_{1}$ & 0 & 0 & 6 & 97 & 193 & 97 & 6 & 6 & 6 \\
\hline$\beta_{0}$ & 0 & 24 & 6 & 1 & 1 & 1 & 1 & 1 & 1 \\
\hline
\end{tabular}

Figure 11. (Color online.) For four disks in a square, the topology changes eight times as the radius varies. Each row and column is unimodal, the maximum of each row is boxed.

for Advanced Study for supporting them in 2010-2011 when much of this work was completed. Stanford supported Jackson Gorham as an undergraduate researcher in 2008 .

\section{REFERENCES}

[1] Allen Hatcher. Algebraic topology. Cambridge University Press, Cambridge, 2002.

[2] Yuliy Baryshnikov, Peter Bubenik, and Matthew Kahle. Min-type Morse theory for configuration spaces of hard spheres. submitted, arXiv:1108.3061, 2011.

[3] S. Torquato and F. H. Stillinger. Jammed hard-particle packings: From Kepler to Bernal and beyond. Rev. Mod. Phys., 82(3):2633-2672, Sep 2010.

[4] Robert Connelly and Walter Whiteley. Second-order rigidity and prestress stability for tensegrity frameworks. SIAM J. Discrete Math., 9(3):453-491, 1996.

[5] Michael Farber. Invitation to topological robotics. Zurich Lectures in Advanced Mathematics. European Mathematical Society (EMS), Zürich, 2008.

[6] Robert Ghrist. Configuration spaces, braids, and robotics. In Braids, volume 19 of Lect. Notes Ser. Inst. Math. Sci. Natl. Univ. Singap., pages 263-304. World Sci. Publ., Hackensack, NJ, 2010.

[7] S.M. LaValle. Planning algorithms. Cambridge University Press, Cambridge, 2006.

[8] Aaron Abrams and Robert Ghrist. Finding topology in a factory: configuration spaces. Amer. Math. Monthly, 109(2):140-150, 2002.

[9] Kenneth Deeley. Configuration spaces of thick particles on a metric graph. Algebraic $\&$ Geometric Topology, 11(2011), 1861-1892.

[10] Persi Diaconis. The Markov chain Monte Carlo revolution. Bull. Amer. Math. Soc. (N.S.), 46(2):179-205, 2009.

[11] K. Böröczky. Über stabile kreis-und kugelsysteme. Ann. Univ. Sci. Budapest Estvss. Sect. Math, 7:79, 1964.

[12] FH Stillinger, H. Sakai, and S. Torquato. Lattice-based random jammed configurations for hard particles. Physical Review E, 67(3):031107, 2003.

[13] A. Donev, S. Torquato, F.H. Stillinger, and R. Connelly. Jamming in hard sphere and disk packings. Journal of applied physics, 95:989, 2004.

[14] B. J. Alder and T. E. Wainwright. Phase transition in elastic disks. Physical Review, 127(2):359-\&, 1962.

[15] Ana C. Ribeiro Teixeira and Daniel A. Stariolo. Topological hypothesis on phase transitions: The simplest case. Phys. Rev. E, 70(1):016113, Jul 2004.

[16] Paolo Grinza and Alessandro Mossa. Topological origin of the phase transition in a model of DNA denaturation. Phys. Rev. Lett., 92(15):158102, Apr 2004.

[17] Luca Angelani, Lapo Casetti, Marco Pettini, Giancarlo Ruocco, and Francesco Zamponi. Topology and phase transitions: From an exactly solvable model to a relation between topology and thermodynamics. Phys. Rev. E, 71(3):036152, Mar 2005.

[18] Michael Farber and Viktor Fromm. Homology of planar telescopic linkages. Algebr. Geom. Topol., 10(2):1063-1087, 2010.

[19] Roberto Franzosi, Marco Pettini, and Lionel Spinelli. Topology and phase transitions I. Preliminary results. Nuclear Physics B, 782(3):189 - 218, 2007. 
[20] Roberto Franzosi and Marco Pettini. Topology and phase transitions II. Theorem on a necessary relation. Nuclear Physics B, 782(3):219 - 240, 2007.

[21] Michael Kastner, Steffen Schreiber, and Oliver Schnetz. Phase transitions from saddles of the potential energy landscape. Physical Review Letters, 99(5):050601, August 2007.

[22] Michael Kastner, Oliver Schnetz, and Steffen Schreiber. Nonanalyticities of the entropy induced by saddle points of the potential energy landscape. Journal of Statistical MechanicsTheory and Experiment, page P04025, April 2008.

[23] L. Caiani, L. Casetti, C. Clementi, and M. Pettini. Geometry of dynamics, Lyapunov exponents, and phase transitions. Physical Review Letters, 79(22):4361-4364, December 1997.

[24] R. Franzosi, L. Casetti, L. Spinelli, and M. Pettini. Topological aspects of geometrical signatures of phase transitions. Physical Review E, 60(5):R5009-R5012, November 1999.

[25] L. Casetti, E. G. D. Cohen, and M. Pettini. Topological origin of the phase transition in a mean-field model. Physical Review Letters, 82(21):4160-4163, May 1999.

[26] L. Casetti, E. G. D. Cohen, and M. Pettini. Exact result on topology and phase transitions at any finite N. Physical Review E, 65(3):036112, March 2002.

[27] R. Franzosi, M. Pettini, and L. Spinelli. Topology and phase transitions: Paradigmatic evidence. Physical Review Letters, 84(13):2774-2777, March 2000.

[28] G. Henkelman, B.P. Uberuaga, and H. Jónsson. A climbing image nudged elastic band method for finding saddle points and minimum energy paths. The Journal of Chemical Physics, 113:9901, 2000.

[29] J. Milnor. Morse theory. Based on lecture notes by M. Spivak and R. Wells. Annals of Mathematics Studies, No. 51. Princeton University Press, Princeton, N.J., 1963.

[30] Y. Yao, J. Sun, X. Huang, G.R. Bowman, G. Singh, M. Lesnick, L.J. Guibas, V.S. Pande, and G. Carlsson. Topological methods for exploring low-density states in biomolecular folding pathways. The Journal of chemical physics, 130:144115, 2009.

[31] Frederick R. Cohen. Introduction to configuration spaces and their applications. In Braids, volume 19 of Lect. Notes Ser. Inst. Math. Sci. Natl. Univ. Singap., pages 183-261. World Sci. Publ., Hackensack, NJ, 2010.

[32] A. Jon Berrick, Frederick R. Cohen, Elizabeth Hanbury, Yan-Loi Wong, and Jie Wu, editors. Braids, volume 19 of Lecture Notes Series. Institute for Mathematical Sciences. National University of Singapore. World Scientific Publishing Co. Pte. Ltd., Hackensack, NJ, 2010. Introductory lectures on braids, configurations and their applications, Papers from the International Conference held at the National University of Singapore, Singapore, June 25-29, 2007.

[33] Robin Forman. A user's guide to discrete Morse theory. Sém. Lothar. Combin., 48:Art. B48c, $35,2002$.

[34] V. Gershkovich and H. Rubinstein. Morse theory for Min-type functions. Asian J. Math., 1(4):696-715, 1997.

[35] Mark Goresky and Robert MacPherson. Stratified Morse theory, volume 14 of Ergebnisse der Mathematik und ihrer Grenzgebiete (3) [Results in Mathematics and Related Areas (3)]. Springer-Verlag, Berlin, 1988.

[36] Congming Jin. Stability, accuracy and cost of NEB and string methods. Commun. Comput. Phys., 2(6):1220-1243, 2007.

[37] S. Torquato and Y. Jiao. Robust algorithm to generate a diverse class of dense disordered and ordered sphere packings via linear programming. Physical Review E, 82(6):061302, 2010.

[38] Persi Diaconis, Gilles Lebeau, and Laurent Michel. Geometric analysis for the Metropolis algorithm on Lipschitz domains. Invent. Math., 185(2):239-281, 2011.

[39] Persi Diaconis, Gilles Lebeau, and Laurent Michel. Gibbs/Metropolis algorithms on a convex polytope. To appear in Math. Zeit., 2011. 
Dept. of Mathematics, Stanford University

E-mail address: <gunnar@math.stanford.edu>

Dept. of Statistics, Stanford University

E-mail address: <jacksongorham@gmail.com>

Dept. of Mathematics, The Ohio State University

E-mail address: <mkahle@gmail.com>

School of Mathematics, Institute for Advanced Study

E-mail address: <jkmason@math.ias.edu> 